\title{
Study of Intelligent Optical Network Technology in Power Communication Network
}

\author{
Feng Wang ${ }^{\text {a }}$, Ning Yu, Dong Wang and Yang Li \\ State Grid Information \&Telecommunication Company of SEPC, Taiyuan 030001,China \\ a5333666@126.com
}

Keywords: ASON, transmission system, power communications network, MSTP.

\begin{abstract}
In this paper, we introduced the ASON technology and the development, have analyzed its architecture characteristic and the function characteristic, and with transmission systems and so on WDM, MSTP, RPR has carried on the comparison. Has obtained the ASON technology for the electric power communications network skeleton line ideal transmission system technology conclusion; elaborated the intelligent light networking survivability and the existence question. Through introduced the Wuhan Electric power company electric power correspondence transmission network before introducing the ASON technology the transmission network present situation, the full use existing optical fiber resources, contrast each factory the equipment, sets up the Wuhan area backbone transmission network, has completed the Wuhan area intelligence light ring net's design.
\end{abstract}

\section{Introduction}

With the development of communication technology, electric power communication is also developed rapidly. With the requirement of all kinds of service, especially the increase of Broad Band, how to build an advanced, steady, reliable transmission network is a very important task in the future.

Compared with the old optical transmission network, Automatically Switched Optical Network (ASON) is more reliable, flexible and efficient. [1] It can offer dynamic configuration for network. It improves the efficiency of network and the ability of rapid protection and restoration, and it supports business needs of different levels of service, and it can automatically discover network resources and better meet IP transmission. [2] ASON currently represents the new direction of optical transmission network development; the telecommunications operators will apply ASON on the network on a larger scale in recent years.

\section{The feasibility study}

This paper is committed to research the appliance of Automatically Switched Optical Network (ASON) in the electric power fiber communication network Traditional electric power fiber communication network mainly for phonetic business design, adopts MSTP based on SDH, [3] the main topological structure is linear and ring, The changes of network topology can't be reflected to the network management in time, poor network expansibility, low bandwidth utilization and so on o With the huge IP data operation is dominating in communication operation bearing the weight of electric power fiber communication network o In order to satisfy the fleetly developing requirement of data operation, the traditional communication network should be upgraded o And now ASON technology already been accepted become the next fiber communication network develop orientation by specific advantage. The relation of physical element and 155/622H equivalent is shown in Table 1.

Table 1 The relation of physical element and 155/622H equivalent

\begin{tabular}{ccc}
\hline Numble & physical element & The number of $155 / 622 \mathrm{H}$ \\
\hline 1 & OptiX 10G & 4 \\
2 & OptiX 2500+ & 3 \\
3 & OptiX 155/622 & 2 \\
4 & OptiX Metro 500 & 1 \\
5 & OptiX OSN9500 & 1 \\
\hline
\end{tabular}




\begin{tabular}{lll}
\hline 6 & OptiX OSN 3500 & 20 \\
7 & OptiX OSN 2500 & 4.5 \\
8 & OptiX 155/622H & 3.5 \\
9 & OptiX OSN 1500 & 1.5 \\
\hline
\end{tabular}

Which, PLC Power Line Communication, 230M data radio transmitted rate is low, defects and poor anti-interference ability, but by way of fiber optic transceivers to connect the low transmission rate, RS-232 bus cable and power cable laying prone with the path of electromagnetic interference generated other defects, GPRS the same way there is a low transmission rate, and take the initiative in hiring public network without problems. ASON network model is shown in Fig. 1.

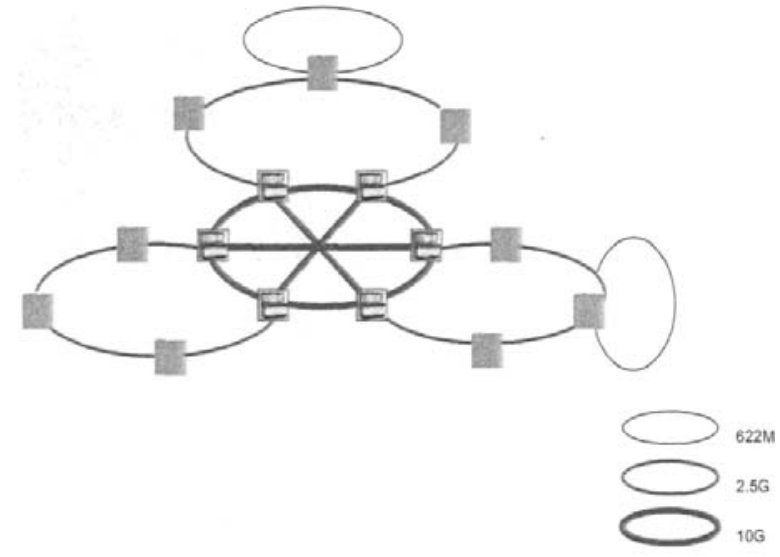

Fig. 1 ASON network model

\section{Survivability of intelligent optical network technology}

According to the practice complexion of fiber communication network of the electric power system, we analysis the trait of electric power and the requirement of consumer operation researching the problems and present situation of MSTP technology, researching the market background of ASON technology lead-in, and the structure, related interface and agreements of ASON technology discussing tactic technology and design application of ASON lead-in fiber communication network. That is [4]:

$$
L 1=\frac{P_{S}-P_{R}-P_{P}-2 A_{C}-M_{C}}{\alpha_{f}+\alpha_{s}}
$$

Where,

$P_{S^{--}}$Optical power $(\mathrm{dBm})$;

$P_{R^{-}-\text {Sensitivity }}(\mathrm{dBm}) ;(\mathrm{BER} \leqslant 10 \mathrm{E}-12)$

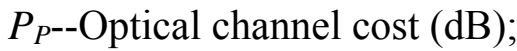

$1.31 \mu \mathrm{m} \mathrm{af}=0.36 \mathrm{~dB} / \mathrm{km}$

$1.55 \mu \mathrm{m}$ af $=0.22 \mathrm{~dB} / \mathrm{km}$

Each connector $A_{C}=0.5 \mathrm{~dB}$

Optical fiber fusion joint average attenuation as $=0.055 \mathrm{~dB} / \mathrm{km}$

Optical fiber rich degrees $M_{C}=3 \mathrm{~dB}$

Power communication systems, assume the power of' main station and between the power system secondary equipment remote monitoring, remote communication, remote debugging, remote control information transmission function the grid resource scheduling work is exceptionally important. How to meet the communication system, power system reliability, high standardized, high-bandwidth nature of investment and economic requirements, is the present and for the next quite a long time now, the face and resolve important issues. [5] In this paper, we are a new means of communication $3 q$ and optical fiber communication in the technical characteristics, reliability, construction costs were compared. We concluded that optical communications is the future direction of development of electric power communication system. 
Table 2 Optical interface board type

\begin{tabular}{cccc}
\hline $622 \mathrm{M}$ & SL4 & S-4.1/4.2 & MTRJ \\
\hline $622 \mathrm{M}$ & SD4 & S-4.1 & SC \\
$2.5 \mathrm{G}$ & SL16A & S-16.1/L-16.1/L-16.2/L-16.2Je & LC \\
$2 \mathrm{M}$ & PQ1 & SMB & $63 * 2 \mathrm{M}$ \\
S164 & $8 / 11$ & $1-9953280 \mathrm{bit}$ & \\
S116 & $6-8$ & $1 * 24888320 \mathrm{bit}$ & \\
\hline
\end{tabular}

\section{Networking scheme of intelligent optical network}

Combined the ASON technology theoretical knowledge and engineering design It's in deep researched that the ASON technology lead-in electric power system, the subject is providing theory and technology for alternation of electric power fiber communication network with better economic and application value. Networking scheme of intelligent optical network is shown in Fig. 2.

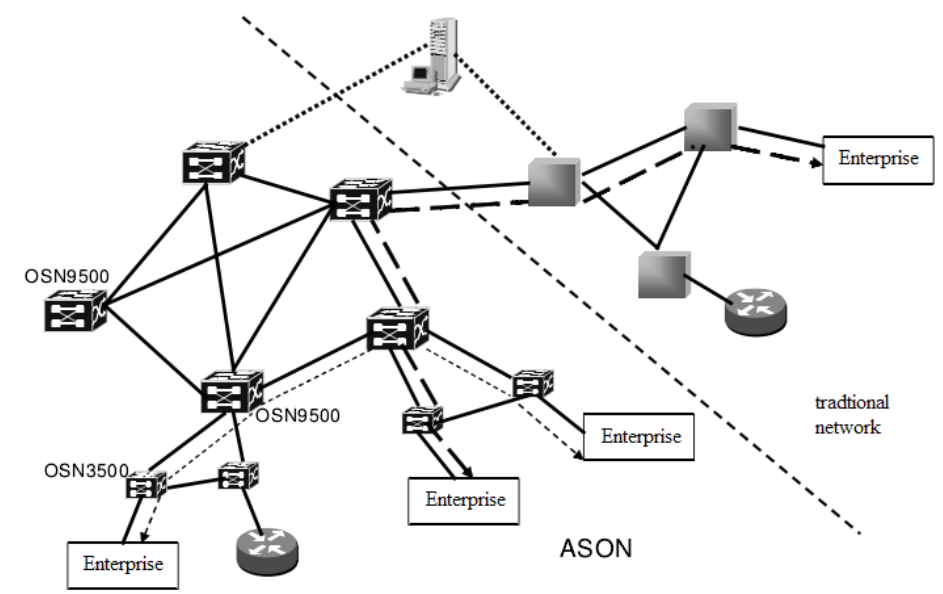

Fig. 2 Networking scheme of intelligent optical network

In this paper, we further discussed the communication optical fiber communication system applications in power feasibility study in electric power communication network, power distribution communication network, respectively, in what light the most appropriate means of communication.

In this paper, optical communication system applications in power feasibility study in electric power communication network, power distribution communication network, respectively, in what light the most appropriate means of communication.

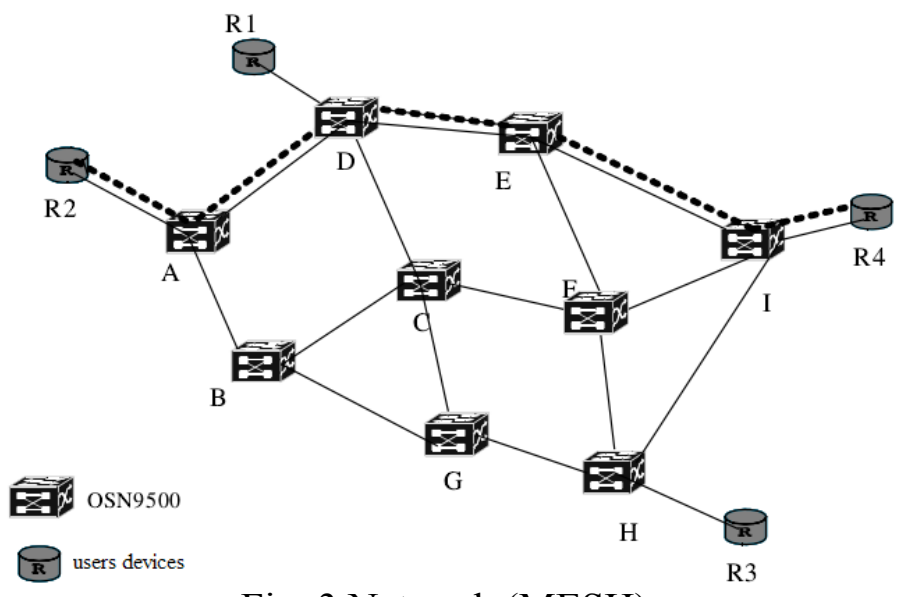

Fig. 3 Network (MESH)

We passed on the existing access to a large number optical communications business of DWDM, OTN, ASON, EPON and other technical characteristics, to build the net maturity for analysis and reference to large network telecommunications operator case. Come the existing power dispatching network and power distribution network communication using optical fiber communication can, and draw a conclusion. GPON compare with EPON is shown in Table 3. 


\begin{tabular}{ll}
\hline & P2MP \\
\hline GPON & EPON \\
ITU.T & IEEE \\
$2.488 \mathrm{G} / 1.244 \mathrm{G}$ & $1.25 \mathrm{G} / 1.25 \mathrm{G}$ \\
$1: 64 \sim 1: 128$ & $1: 16 \sim 1: 32$ \\
ATM,Ethemet,TDM & Ethemet \\
$92 \%$ & $72 \%$ \\
Very good,including Ethemet,TDM,ATM & Good, only ethemet \\
Class A/B/C & Px10/Px20 \\
EqD & RTT \\
TDM over Ethemet (PWE3,CESoEthemet) or native & TDM over Ethemet (PWE3,CESoEthemet) or native \\
TDM) & TDM) \\
OMCI & \\
\hline
\end{tabular}

In electric power communication network in the networking and telecommunications operators are now fully consistent network of electric power network can be applied to more higher rate DWDM wavelength system, the future system can transition to the OTN network.

Communication in power distribution network, the widespread use of telecommunications operators EPON broadband access communication system transplant, is a very good choice. EPON technology high network security, expansion of convenient, easy to maintain, but also save construction costs, possible future transition to GPON system network.

\section{Conclusions}

Based on the concept of intelligent optical network and system structure, intelligent optical network routing automatic discovery and mechanism for protection and restoration were discussed, and construction planning of ASON in power communication network is put forward according to the actual situation of electric power communication network.

\section{References}

[1] R.F.Vazquez Z, J.Feyen. Assessment of the Performance of a Distributed Code in Relation to the ETp Estimates. Water Resources Management, 2012, (16): 329-350.

[2] Thorsten Graef, Ulrich Steidl, Wolfgang Nedbal, Ulrich Rohr, Roland Fenk,Rainer Haas, Ralf Kronenwett. Use of RNA to inhibit integrin subunit a V mediated angiogenesis. Angiogenesis, 2010, (02): 361-372.

[3] Andreas Kirstadter, Andreas Iselt, Achim Autenrieth. DominicA.Schupke, Robert Prinz, Bernhard Edmaier. Business Models for Next Generation Transport Networks. Photonic Network Communications, 2013, (11): 283-296.

[4] Zhou Tao, Guo Lei,=Yu Hong-fang, et a1. A routing algorithm with no-shared-risk for WDM optical networks. Journal of University of Electronics Science and Technology of China, 2006, 8(4): 440-443.

[5] Yu Hong-fang, Wen Haibo, Wang Sheng, et al. Shared-path protection algorithm with differentiated reliability in meshed WDM networks. Journal of Electronics \& Information Technology, 2009, 27(8): 1295-1298. 